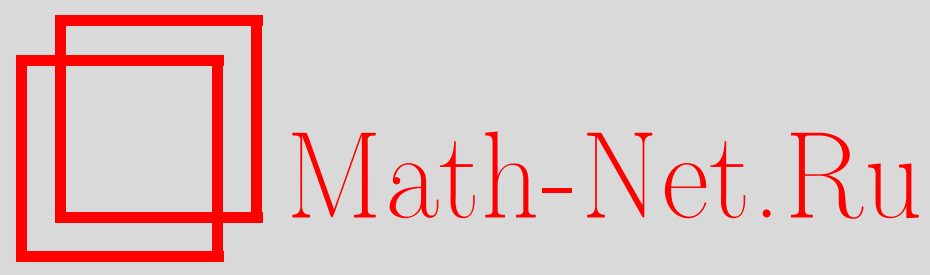

У. А. Розиков, Случайные блуждания в случайных средах на метрических группах, Матем. заметки, 2000, том 67, выпуск 1, 129-135

DOI: https://doi.org/10.4213/mzm820

Использование Общероссийского математического портала Math-Net.Ru подразумевает, что вы прочитали и согласны с пользовательским соглашением http://www . mathnet.ru/rus/agreement

Параметры загрузки:

IP : 54.224 .135 .184

26 апреля 2023 г., 14:39:20

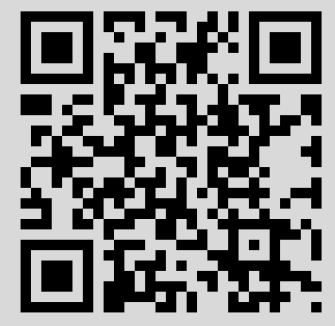




\title{
СЛУЧАЙНЫЕ БЛУЖДАНИЯ В СЛУЧАЙНЫХ СРЕДАХ НА МЕТРИЧЕСКИХ ГРУППАХ
}

\author{
У. А. Розиков
}

Рассматриваются случайные блуждания в случайной среде на счетных группах $\mathrm{c}$ метрикой, когда размеры скачков блуждающей частицы ограничены. Вероятности перехода такого случайного блуждания из точки $x \in G$ (где $G$ - рассматриваемая группа) определяется вектором $p(x) \in \mathbb{R}^{|W|}$ (где $W \subset G$ - фиксировано и $|W|<\infty$ ). Предполагается, что совокупность $\{p(x), x \in G\}$ есть совокупность независимых одинаково распределенных случайных векторов. Для такого случайного блуждания найдено достаточное условие невозвратности. В качестве примера рассмотрены группы $Z^{d}$, свободные группы и группа свободного произведения конечного числа циклических групп второго порядка.

Библиографоия: 15 названий.

1. Введение. Случайные блуждания в случайных средах возникли в различных задачах кристаллографии [1], физики металлов [2], биофизики [3]. За последние два десятилетия теория случайных блужданий в случайных средах превратилась в один из наиболее бурно развиваюшихся разделов теории вероятностей. Простейшее одномерное случайное блуждание в случайных средах, когда движущаяся частица за единицу времени может переместиться только на один шаг вправо или влево, было рассмотрено в работе $\Phi$. Соломона [4]. Он вьписал критерий возвратности для такой модели случайного блуждания. Я.Г. Синаем [5] было доказано, что при условии возвратности предельное поведение случайного блуждания $\{x(t), t=0,1,2, \ldots\}$ отлично от классического, и, в частности, случайная величина $x(t) \ln ^{-2} t$ имеет предельное распределение при $t \rightarrow+\infty$. При тех же условиях П. Дехевелс и П. Ревез [6] исследовали асимптотические свойства этого процесса с вероятностью 1 . Более сложные случайные блуждания в случайных средах на $\mathbb{Z}$, когда размеры скачков блуждающей частицы ограничены, были изучены в работах Э. Кея [7], А.В. Летчикова [8], [9]. В работах Синая, Аншелевича, Ханина [10] было рассмотрено случайное блуждание в симметрических случайных средах на $Z^{d}$. Лоулер [11] доказал центральную предельную теорему (ц.п.т.) для таких блужданий при некоторых условиях на среды. А. Астраускас, Д. Сургайлис [12] доказали ц.п.т. в случае мартингальности среды на $Z^{d}$.

В настоящей работе мы изучаем предельное поведение траектории случайного блуждания в случайной среде на счетных метрических группах. Пункт 2 содержит основные понятия и постановку задачи. В пункте 3 доказьвается одна теорема (теорема 3 ) и в 
пункте 4 изучаются различные случайные блуждания в случайных средах; для каждого случая проверяются условия теоремы 3 .

Автор выражает благодарность Н. Н. Ганиходжаеву за полезные замечания и внимание к работе.

2. Основные понятия и постановка задачи. Пусть $(G, \circ, \rho)$ - счетная, метрическая группа с бинарной операцией о, где $\rho$ - некоторая метрика.

Пусть $x_{0} \in G$ фиксированный элемент и $W\left(x_{0}\right)$ - фиксированное, конечное подмножество $G$ такое, что $x_{0} \in W\left(x_{0}\right)$.

Предположим, что $\theta_{x}: G \rightarrow G$ определено следующим образом: $\theta_{x} y=x \circ y$. Обозначим $W(x)=\theta_{x} W\left(x_{0}\right)$.

Пусть $H$ множество векторов $p=\left(p_{u}, u \in W\left(x_{0}\right)\right) \in \mathbb{R}^{\left|W\left(x_{0}\right)\right|}$ таких, что для всех $u \in W\left(x_{0}\right), p_{u} \geq 0, \sum_{u \in W\left(x_{0}\right)} p_{u}=1$. Обозначим через $B$ множество борелевских подмножеств $H$. Положим, что на $H$ задано некоторое распределение вероятностей $\mu$.

Пространством случайных сред $\left(\Omega, F_{\Omega}, \mathrm{P}\right)$ назовем прямое произведение: $\left(\Omega, F_{\Omega}, \mathrm{P}\right)=\prod_{x \in G}(H, B, \mu)$, а средой - $A$-элемент множества $\Omega$. Таким образом, $A=\{p(x) \in H, x \in G\}$ есть совокупность $H$-значных векторов.

Пусть $A=\{p(x) \in H, x \in G\}$ - фиксировано.

ОПРЕДЕЛЕНИЕ 1. Случайным блужданием в фиксированной среде $A$ с начальным состоянием $x_{0}$ называется однородная по времени цепь Маркова с временньп пространством $T=\{0,1,2, \ldots\}$, фазовьм пространством $G$ и матрищей переходных вероятностей $M=\left\{m_{x y}, x, y \in G\right\}$, элементы которой определяются по формуле:

$$
m_{x y}= \begin{cases}p_{y}(x), & \text { если } y \in W(x), \\ 0 & \text { в остальных случаях }\end{cases}
$$

где $\left\{p(x)=\left(p_{y}(x), y \in W(x)\right), x \in G\right\}$.

Обозначим через $X=G^{T}$ множество всевозможных траекторий случайного блуждания в среде $A$. Пусть $F_{X}-\sigma$-алгебра, порожденная цилиндрическими подмножествами $X$.

Обозначим через $\mathrm{P}_{A, x_{0}}$ вероятность в $\left(X, F_{X}\right)$, соответствующую случайному блужданию в среде $A$, начинающемуся в точке $x_{0}$.

ОПРЕДЕЛЕНИЕ 2. Случайным блужданием в случайной среде будем назьвать случайный процесс $X(t)=X(t, \omega), t \in T$, определенный на измеримом пространстве $\left(\Omega_{1}, F\right)=\left(X \times \Omega, F_{X} \times F_{\Omega}\right)$ равенством

$$
X(t, \omega)=x_{t}, \quad \omega=(x, A) \in \Omega_{1},
$$

где $x=\left(x_{t}, t \in T\right) \in X$, а вероятность $\mathrm{P}_{x_{0}}$ на $\left(\Omega_{1}, F\right)$ однозначно определяется своими значениями на множествах $B=B_{1} \times B_{2}, B_{1} \in F_{X}, B_{2} \in F_{\Omega}$, равными

$$
\mathrm{P}_{x_{0}}(B)=\int_{B_{2}} \mathrm{P}_{A, x_{0}}\left(B_{1}\right) \mathrm{P}(d A) .
$$


В данной работе мы изучаем предельное поведение траектории такого случайного блуждания в случайных средах. Эта задача сложная и полно решается только относительно частньх случаев $(G, \circ, \rho)$.

В п. 3 мы выделим класс сред (периодических), для которых эта задача решается для достаточно широких классов $(G, \circ, \rho)$.

\section{3. Предельное поведение траектории в периодической случайной сре-} де. Изучение рассматриваемой проблемы для некоторых частньх случаев (относительно $(G, \circ, \rho)$ и случайной среды) мы приведем к изучению случайного блуждания $\{\tilde{x}(t), t \in T\}$ в случайной среде на $\mathbb{Z}$, в котором за единицу времени частица может переместиться не более, чем на $b \in \mathbb{N}$ шагов вправо, и не более, чем на $a \in \mathbb{N}$ шагов влево. Вероятности перехода такого блуждания из точки $x \in \mathbb{Z}$ определяются вектором $p(x) \in \mathbb{R}^{a+b+1}$. Предполагается, что последовательность $\{p(x), x \in \mathbb{Z}\}$ есть последовательность независимых одинаково распределенных случайных векторов. Такая модель уже рассматривалась в печати. Э. Кей[7] вьписал для нее критерий возвратности. В статье [9] исследовано асимптотическое поведение возвратного случайного блуждания в случайной среде в смысле сходимостей по вероятности и по распределению. В работе [8] исследованы асимптотические свойства рассматриваемой модели с вероятностью 1 , доказаны принцип инвариантности и закон повторного логарифма для произведения независимых случайных матриц.

Мы используем следующую теорему

ТЕОрема 1 [7], [9]. Справедливы следующие импликаиии:

1) $\lambda_{b}>0 \Longrightarrow P_{0}\left\{\lim _{t \rightarrow+\infty} \tilde{x}(t)=+\infty\right\}=1$,

2) $\lambda_{b}=0 \Longrightarrow \mathrm{P}_{0}\left\{\lim \sup _{t \rightarrow+\infty} \tilde{x}(t)=+\infty \liminf _{t \rightarrow+\infty} \tilde{x}(t)=-\infty\right\}=1$,

3) $\lambda_{b}<0 \Longrightarrow \mathrm{P}_{0}\left\{\lim _{t \rightarrow+\infty} \tilde{x}(t)=-\infty\right\}=1$,

где $\lambda_{b}-$ показатель Ляпунова.

Пусть $\Phi$ - множество отображений $\varphi$ пространства $G$ на $\mathbb{Z}$ со следующими свойствами:

$$
\begin{gathered}
|\varphi(x)| \leq \rho\left(x_{0}, x\right) \quad \forall x \in G, \\
\varphi(W(x))=T_{\varphi(x)}\left(\varphi\left(W\left(x_{0}\right)\right)\right) \quad \forall x \in G,
\end{gathered}
$$

где $T_{z} y=z+y, z, y \in \mathbb{Z}$.

Возникает вопрос: при каких условиях на $(G, \circ, \rho)$, множество $\Phi$ будет пустым множеством? Частичным ответом на этот вопрос будет следующая

Теорема 2. Если $\rho$ конечная (т.е. существует $M>0$ такое, что для любих $x, y \in G$ выполняется $\rho(x, y) \leq M)$, то $\Phi=\varnothing$.

ДокАЗАтЕльство. Так как $\varphi$ отображение на $\mathbb{Z}$, для любого $M>0$ можно найти $x \in G$ такое, что $|\varphi(x)|>[M]+1$. Откуда следует, что не выполняется свойство $(1)$ для $\varphi$ при конечных $\rho$. Теорема доказана. 
Определим на $G$ бинарное отношение $\sim_{\varphi}$, положив $x \sim_{\varphi} y$ для $x, y \in G$ тогда и только тогда, когда $\varphi(x)=\varphi(y)$. Очевидно, что отношение $\sim_{\varphi}$ является отношением эквивалентности и, следовательно, множество $G$ разбивается на классы эквивалентных элементов: все элементы, входящие в один класс, отображаются в один элемент на $\mathbb{Z}$. Обозначим через $G_{u}^{\varphi}$ класс элементов $x$ из $G$, эквивалентных данному элементу $u: x \sim \varphi$ $u$. Для $\forall x \in G, \forall z \in \mathbb{Z}$ обозначим $a_{z}^{\varphi}(x)=\sum_{y \in W(x): \varphi(y)=z} p_{y}(x)$.

ОПРЕДЕЛЕНИЕ 3 . Среду $A=\{p(x), x \in G\} \in \Omega$ назовем $\varphi$-периодической, если для любых $u \sim_{\varphi} v$ имеет место равенство $a_{z}^{\varphi}(u)=a_{z}^{\varphi}(v) ; z \in \mathbb{Z}$.

Теорема 3. Если существует $\varphi \in \Phi$ такое, что $\Omega$ состоит только из $\varphi$-периодических сред, то справедливы следующие импликачии:

(1) 1)" $\lambda_{b} \neq 0 \Longrightarrow \mathrm{P}_{x_{0}}\left\{\lim _{t \rightarrow+\infty} \rho\left(x_{0}, X(t)\right)=+\infty\right\}=1$

2) $\lambda_{b}=0 \Longrightarrow \mathrm{P}_{x_{0}}\left\{\lim \sup _{t \rightarrow+\infty} \rho\left(x_{0}, X(t)\right)=+\infty\right\}=1$,

где $\lambda_{b}-$ показатель Ллпунова, $b=\max \varphi\left(W\left(x_{0}\right)\right)$.

ДОКАЗАТЕЛЬСТВО. В силу условий теоремы и свойств $\varphi$ ясно, что функция $\varphi$ индуцирует случайное блуждание $\{\tilde{x}(t), t \in T\}$ с пространством состояний $\mathbb{Z}$, начальным состоянием 0 и матрицей переходных вероятностей $\widetilde{M}=\left\{m_{x y}, x, y \in \mathbb{Z}\right\}$, элементы которой определяются по следующей формуле:

$$
m_{x y}= \begin{cases}a_{y-x}^{\varphi}(x), & \text { если } y-x \in \varphi\left(W\left(x_{0}\right)\right) \\ 0 & \text { в остальных случаях. }\end{cases}
$$

В силу свойства (1) функции $\varphi$ имеем $|\tilde{x}(t)| \leq \rho\left(x_{0}, X(t)\right) \forall t \in T$. Из последнего неравенства, применяя теорему 1 , получаем 1$)$ и 2 ). Теорема доказана.

Заметим, что в случае конкретного $G$ для применения теоремы 3 необходимо показать существование отображения $\varphi \in \Phi$, для которого выполняется условие теоремы 3 . Эта задача достаточно сложная и неизвестна общая конструкция построения такого отображения (если оно существует).

Заметим, что в силу теоремы 2 мы можем построить достаточно широкий класс метрических пространств, для которых не существует $\varphi \in \Phi$.

В п. 4 мы рассматриваем несколько примеров, где для каждого случая строятся сначала функции $\varphi \in \Phi$ и для построенного $\varphi$ требуется вьполнение условия теоремы 3 .

4. Примеры.

1. Самым простым примером является следующий пример: $G=Z^{d}, d \geq 1$, с обычным сложением + и метрикой

$$
\rho(x, y)=\sum_{i=1}^{d}\left|x_{i}-y_{i}\right|, \quad\left(x=\left(x_{1}, \ldots, x_{d}\right), \quad y=\left(y_{1}, \ldots, y_{d}\right)\right)
$$

Пусть $x_{0}=(0,0, \ldots, 0) \in Z^{d}$ начальное состояние. Функцию $\varphi: Z^{d} \rightarrow \mathbb{Z}$ определим по формуле $\varphi(x)=\varphi\left(x_{1}, \ldots, x_{d}\right)=x_{1}$. Проверим, что $\varphi \in \Phi$ удовлетворяет $(1),(2)$ : $|\varphi(x)|=\left|x_{1}\right| \leq \rho(0, x)=\sum_{i=1}^{d}\left|x_{i}\right| ;(2)$ следует очевидным образом из гомоморфности $\varphi$. 
Относительно $\varphi$ пространство $Z^{d}$ разбивается на классы эквивалентности: $Z_{x}^{d, \varphi}=$ $\left\{y \in Z^{d}: x_{1}=y_{1}\right\}$. Требуется условие $\varphi$-периодичности среды $A$. Тогда будет справедлива теорема 3 .

2. Пусть $G$ - свободная группа с множеством свободных образующих $M$. Бинарная операция о - это написание слова за словом. Метрику определим по формуле $\rho(x, y)=$ $\min \left\{d \mid \exists x=x_{1}, x_{2}, \ldots, x_{d-1}, x_{d}=y\right.$ таких, что для $x_{i}$ и $x_{i+1} \exists a \in M$ такое, что $x_{i}=$ $x_{i+1} a$ либо $\left.x_{i+1}=x_{i} a\right\}$. Последовательность $x_{1}, x_{2}, \ldots, x_{d}$, реализующая указанный минимум, назьвается путем из $x$ в $y$ и обозначается $\pi(x, y)$.

Пусть $e \in G$ единичный элемент этой групшы. Отображение $\varphi: G \rightarrow \mathbb{Z}$ строим конструктивно: зафиксируем $a \in M$. Пусть $\pi_{a}: M \rightarrow\left\{e, a, a^{-1}\right\}$ определяется по формуле:

$$
\pi_{a}(x)=\left\{\begin{array}{l}
x, \text { если } x=a, a^{-1}, \\
e, \text { если } x \in M \backslash\left\{a, a^{-1}\right\} .
\end{array}\right.
$$

Обозначим через $G_{a}$ - группу со свободным образующим $a$. Очевидно, что $G_{a}$ бесконечная циклическая группа. Для $a \in M$ отображение $f_{a}: G \rightarrow G_{a}$ определим следующим образом:

$$
f_{a}(x)=f_{a}\left(x_{i_{1}}^{\varepsilon_{1}} x_{i_{2}}^{\varepsilon_{2}} \ldots x_{i_{n}}^{\varepsilon_{n}}\right)=\pi_{a}\left(x_{i_{1}}^{\varepsilon_{1}}\right) \ldots \pi_{a}\left(x_{i_{n}}^{\varepsilon_{n}}\right),
$$

где $\varepsilon_{i}= \pm 1, i=1,2, \ldots, n$, и $n=|x|$ назьвается длиной слова $x \in G$.

Введем следующие отображения $g_{a}: G_{a} \rightarrow \mathbb{Z}: g_{a}\left(a^{m}\right)=m ; \varphi_{a}: G \rightarrow \mathbb{Z}: \varphi_{a}(x)=$ $g_{a}\left(f_{a}(x)\right)$.

ПРЕДЛОЖЕНИЕ 1. Для любого $а \in M$ справедливы следующие утверждения:

i) $\rho(x, y) \geq\left|\varphi_{a}(x)-\varphi_{a}(y)\right|$;

ii) если $\theta_{x}: G \rightarrow G$ сдвиг (левьй) на $G u T_{n}: \mathbb{Z} \rightarrow \mathbb{Z}$ сдвигна $\mathbb{Z}$, то $\varphi_{a}\left(\theta_{x} y\right)=$ $T_{\varphi(x)} \varphi(y) \quad \forall x, y \in G$.

ДокАЗАтЕЛЬСтво. і) Очевидно, что $f_{a}$ и $g_{a}$ являются гомоморфизмами. Следовательно, $\varphi_{a}$ является гомоморфизмом. В силу гомоморфности $\varphi_{a}$ имеем:

$$
\begin{aligned}
& \varphi_{a}(x)=\varphi_{a}\left(x_{i_{1}}^{\varepsilon_{1}} x_{i_{2}}^{\varepsilon_{2}} \ldots x_{i_{n}}^{\varepsilon_{n}}\right)=\varphi_{a}\left(x_{i_{1}}^{\varepsilon_{1}} x_{i_{2}}^{\varepsilon_{2}} \ldots x_{i_{n-1}}^{\varepsilon_{n-1}}\right)+\varphi_{a}\left(x_{i_{n}}^{\varepsilon_{n}}\right), \\
& \varphi_{a}(x)= \begin{cases}\varphi_{a}\left(x x_{i_{n}}^{-\varepsilon_{n}}\right), & \text { если } x_{i_{n}}^{\varepsilon_{n}} \neq a, a^{-1}, \\
\varphi_{a}\left(x x_{i_{n}}^{-\varepsilon_{n}}\right)+1, & \text { если } x_{i_{n}}^{\varepsilon_{n}}=a, \\
\varphi_{a}\left(x x_{i_{n}}^{-\varepsilon_{n}}\right)-1, & \text { если } x_{i_{n}}^{\varepsilon_{n}}=a^{-1} .\end{cases}
\end{aligned}
$$

Из последних равенств заметим, что соседние точки пути $\pi(x, y)$ отображением $\varphi_{a}$ переводятся либо в одну точку, либо в соседние точки на $\mathbb{Z}$, откуда следует i).

ii) Непосредственно следует в силу гомоморфности отображения $\varphi_{a}$. Предложение доказано.

Из предложения 1 следует, что $\varphi_{a} \in \Phi$ для любого $a \in M$. Обозначим $H_{a}=\{x \in$ $\left.G: \varphi_{a}(x)=e\right\}$. Ясно, что $H_{a}$ является нормальным делителем. Условие $\varphi_{a}$-периодичности случайной среды в этом случае совпадает с $H_{a}$-периодичностью (т.е. $a_{z}^{\varphi}(y)=$ $a_{z}^{\varphi}\left(\theta_{x} y\right)$, если $\left.y \in G, x \in H_{a}, z \in \mathbb{Z}\right)$. При этих условиях справедлива теорема 3 . 
Заметим, что свободная группа $G$ определяется заданием мошности множества $M$ и не зависит ни от каких индивидуальных свойств элементов этого множества.

Известно, что если $M$ - конечное или счетное множество, то $G$ будет счетным, если $M$ несчетно, то и $G$ будет несчетным [13]. В случае $|M|<\infty$ граф этой группы будет деревом Кэли порядка $2|M|-1$ [14], [15].

В примерах 1, 2 построенные отображения являются гомоморфизмами. Из этого свойство (2) функции $\varphi$ следовало непосредственно. В следующем примере построим отображение, не являющееся гомоморфизмом, но принадлежащее $\Phi$.

3. Пусть $G_{k}, k \geq 1$, свободное произведение $k+1$ циклических групп второго порядка с образующими $a_{1}, a_{2}, \ldots, a_{k+1}$ соответственно. Бинарная операция и метрика определяются так же, как в примере 2. Пусть $e$ - единичный элемент. Для фиксированньх $i, j=1,2, \ldots, k+1(i \neq j)$ введем следующие отображения.

a) Отображение $\pi_{i j}:\left\{a_{1}, \ldots, a_{k+1}\right\} \rightarrow\left\{e, a_{i}, a_{j}\right\}$, которое задается как

$$
\pi_{i j}\left(a_{m}\right)=\left\{\begin{array}{lll}
a_{m}, & \text { если } m=i, j, \\
e, & \text { если } m \neq i, j .
\end{array}\right.
$$

b) Пусть $G_{i j}$ - свободное произведение циклических групा $\left\{e, a_{i}\right\},\left\{e, a_{j}\right\}$. Отображение $f_{i j}: G_{k} \rightarrow G_{i j}$ определяется равенством $f_{i j}=f_{i j}\left(a_{i_{1}} \ldots a_{i_{n}}\right)=\pi_{i j}\left(a_{i_{1}}\right) \ldots \pi_{i j}\left(a_{i_{n}}\right)$.

c) Отображение $g_{i j}: G_{i j} \rightarrow \mathbb{Z}$ определяется равенством

$$
g_{i j}(y)= \begin{cases}|y|, & \text { если } y=a_{i} a_{j} a_{i} a_{j} \ldots, \\ -|y|, & \text { если } y=a_{j} a_{i} a_{j} a_{i} \ldots, \\ 0, & \text { если } y=e .\end{cases}
$$

d) Отображение $\varphi_{i j}: G_{k} \rightarrow \mathbb{Z}$ определим по формуле: $\varphi_{i j}(x)=g_{i j}\left(f_{i j}(x)\right)$. Очевидны следующие свойства функции $\varphi_{i j}$.

ПРЕДЛОЖЕНИЕ 2. Для любы $i, j=1,2, \ldots, k+1(i \neq j)$ справедливы следующие утверждения:

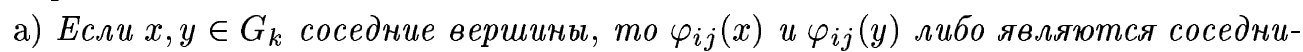
мu, либо $\varphi_{i j}(x)=\varphi_{i j}(y)$;

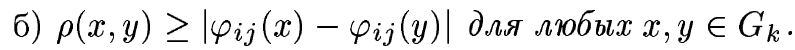

Из предложения 2 следует, что $\varphi_{i j} \in \Phi, i, j=1, \ldots, k+1(i \neq j)$. Таким образом, в этом случае можно доказать теорему 3 в случае $\varphi_{i j}$-периодичности случайных сред.

\section{СПИСОК ЦИТИРОВАННОЙ ЛИТЕРАТУРЫ}

[1] Темкин Д.Е. К теории бездиффузионного роста кристаллов // Кристаллография. 1969. T. 14. № 3. C. 423-430.

[2] Bernasconi J., Schnerider W.R., Wyss W. Diffusion and hopping conductivity in disordered one-dimensional lattice systems // Zeit. Phys. B. 1980. V. 37. P. 175-184.

[3] Alexander S., Bernasconi J., Schneder W. R., Orbach K. Excitation dynamics in random one-dimensional system // Rev. Modern. Phys. 1981. V. 53. № 2. P. 175-198. 
[4] Solomon F. Random walks in a random environment // Ann. Prob. 1975. V. 3. № 1. P. 1-31.

[5] Синай Я.Г. Предельное поведение одномерного случайного блуждания в случайной среде // Теория вероятн. и ее применения. 1982. Т. 27. № 2. С. 247-258.

[6] Deheuvels P., Revesz P. Simple random walk on the line in random environment // Prob. Th. Rel. Fields. 1986. V. 72. № 2. P. 215-230.

[7] Key E. Recurrence and transience criteria for random walk in a random environment // Ann. Prob. 1984. V. 12. № 2. P. 529-560.

[8] Летчиков А. В. Асимптотические свойства одномерных случайных блужданий в случайной среде с вероятностью 1 // Матем. сб. 1991. Т. 182. № 12. С. 1710-1728.

[9] Летчиков А. В. Предельная теорема для возвратного случайного блуждания в случайной среде // Докл. АН СССР. 1989. Т. 302. №1. С. 25-28.

[10] Anshelevich V.V., Khanin K. M., Sinai Ya. G. Symmetric random walks in random environments // Comm. Math. Phys. 1982. V. 85. № 3. P. 449-470.

[11] Lawler G.F. A discrete stochastic integral inequality and balanced random walk in a random environment // Duke Math. J. 1983. V. 50. № 4. P. 1261-1274.

[12] Астраускас А., Сургайлис Д. Предельные теоремы для случайного блуждания в случайной среде // Литовский матем. сб. 1985. Т. 256. № 2. С. 12-27.

[13] Курош А. Г. Теория групп. М.: Наука, 1967.

[14] Ганиходжаев Н. Н. Групповое представление и автоморфизмы дерева Кэли // Докл. АН РУз. 1994. № 4. С. 3-5.

[15] Магнис В., Каррас А., Солитэр Д. Комбинаторная теория групп. М.: Наука, 1974.

Институт математики им. В. И. Романовского

E-mail: root@im.tashkent.su. 